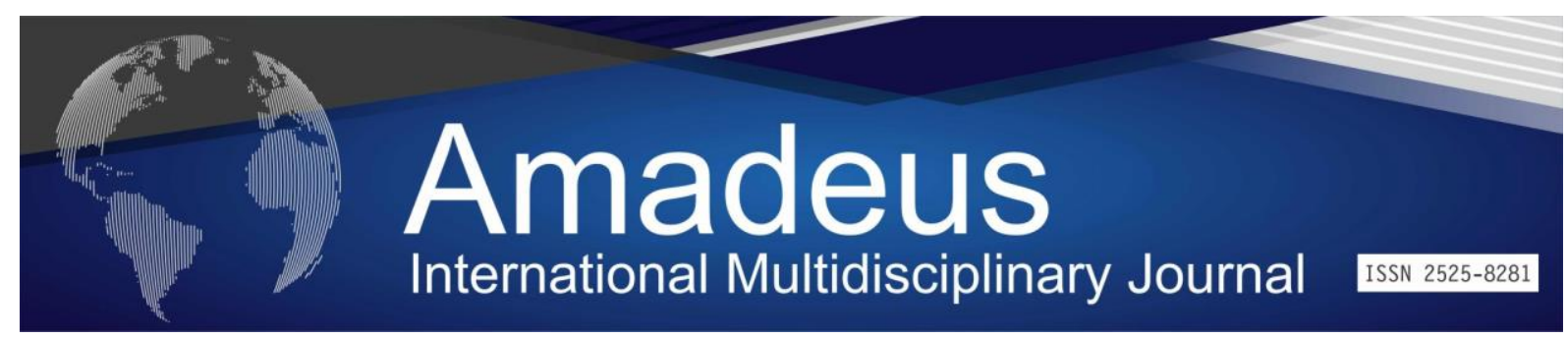

DOI: 10.14295/aimj.v5i9.131

\title{
Biopharmaceuticals: A New Alternative in Cancer Treatment?
}

João Francisco Lucena Franco Filho ${ }^{l}$;

José Leonardo Gomes

Coelho ${ }^{2}$;

Carmelita Maria Silva

Sousa $^{3}$;

Elis Augusta Moura Dias

Fernandes $^{4}$;

Rivaniery Teles Braga

Emídio de Brito

Willma José de Santana
Abstract: The purpose of this comment is to report the technological advancement of biopharmaceuticals in the fight against cancer. This is a comment, an investigative text from the specialized literature on the subject, selected scientific articles were used in the years 2016 to 2020 . In English and Portuguese. The following were listed: biopharmaceuticals, cancer, Innovation. This comment is expected to describe the main innovations that modern medicine can bring in the fight against cancer.

Keywords: Biopharmaceuticals; Cancer; innovation.

\section{Biofármacos: Uma Nova Alternativa no Tratamento do Câncer?}

Resumo: O objetivo deste comentário é relatar o avanço tecnológico dos biofármacos no combate ao câncer. Trata-se de um comment, um texto investigativo da literatura especializada sobre a temática, foram utilizados artigos científicos selecionados nos anos de 2016 a 2020.Nos idiomas inglês e português. Foram elencados os seguintes descritos: biofármacos, câncer, Inovação. Espera-se com este comentário descrever as principais inovações que a medicina moderna pode trazer no combate ao câncer.

Palavras-chaves: Biofármacos; Câncer; inovação.

\footnotetext{
${ }^{1}$ Acadêmico de Farmácia do Centro Universitário de Juazeiro do Norte - UNIJUAZEIRO.

Email: joaofilholucena@gmail.com;

${ }^{2}$ Acadêmico de Farmácia do Centro Universitário de Juazeiro do Norte - UNIJUAZEIRO. ORCID: http://orcid.org/00000001-6028-0807. Email: leonardo-coelho-10@hotmail.com;

${ }^{3}$ Mestranda em Políticas Públicas pela Universidade Athenas College. ORCID http://orcid.org/0000-0002-2288-5554. Email: carmelitasilva11@ @otmail.com;

${ }^{4}$ Acadêmica de Farmácia do Centro Universitário de Juazeiro do Norte - UNIJUAZEIRO.

Email: elisaugustamoura@gmail.com;

${ }^{5}$ Acadêmica de Farmácia do Centro Universitário de Juazeiro do Norte - UNIJUAZEIRO.

Email: rivaniery1@ hotmail.com;

${ }^{6}$ Pós Doutoranda em Ciências da Saúde - FMABC, Doutora em Ciências Biológicas pela UFPE e Docente do Centro Universitário de Juazeiro do Norte - UNIJUAZEIRO e Faculdade de Tecnologia - FATEC - CARIRI. ORCID http://orcid.org/0000-0003-2733-2892. Email: wjsantana@ hotmail.com.
} 


\section{Introdução}

Desde 1928 quando Alexander Fleming acidentalmente descobriu a penicilina, o primeiro antibiótico, os medicamentos não pararam de evoluir, tornando-se mais eficazes, menos agressivos/invasivos e com menos efeitos adversos (Oliveira; Silva, 2018). Todavia, a grande inovação da medicina moderna são os medicamentos biológicos, tornando assim uma grande possibilidade de serem usados no futuro para o tratamento de doenças.

Os Medicamentos biológicos ou biofármacos são medicamento produzidos por compostos químicos de células vivas, via biotecnológicos. Sendo assim por serem moléculas complexas, não é possível obterem copias idênticas do mesmo (Brasil, 2010). Existem algumas doenças na atualidade que são tratadas com biofármacos, como: Mal de Alzheimer, Câncer de Mama, Câncer renal, diabetes, hepatite, leucemia e entre outros (Brasil, 2018).

Câncer é um termo genérico que se caracteriza por um crescimento descontrolado de células heterógenas, que aleatoriamente pela replicação celular fogem parcial ou totalmente do padrão genético, com efeitos agressivos sobre o organismo, podendo afetá-lo em qualquer parte (Brasil, 2011; Who, 2016).

Nos últimos tempos, pesquisadores e cientistas enfrentam a batalha de desenvolver interações que busque novos estímulos de respostas imunes ou a potencialização imunológica contra células tumorais. Estas intervenções são classificadas como imunoterapias, tendo interação entre antígeno-anticorpo resultado de mecanismos de defesa celulares. Sendo assim, a imunoterapia anticâncer evoluiu de uma promissora opção terapêutica para uma realidade clínica (Galluzzi et al, 2014; Brasil, 2017). Pesquisadores afirmam que os biofármacos podem diminuir os efeitos colaterais no tratamento do câncer se comparado a quimioterapia clássica (Ferreira et al, 2014).

Essa classe de medicamento, tem muito a ser desenvolvido, possui uma maior complexidade em seu desenvolvimento. Esse processo produtivo possui várias etapas na sua produção. Desde da linhagem celular, cultivo celular, purificação e filtração até chegar na formulação e envase (Castilho, 2010).

\section{Método}

Trata-se de um comment (Delmondes; Coelho; Neves; Santana, 2020) sobre a temática de biofármacos como alternativa no tratamento do câncer. Foram selecionados artigos 
científicos dos anos de 2016 a 2020. Nos idiomas inglês e português. Excluindo artigos de língua espanhola.

(I) a avaliação foi constituída juntamente com a reflexão sobre o método de fabricação dos biofármacos e em seguida demonstrar os pontos positivos desse medicamento.

(II) foi realizado um analise crítica dos variados exemplos de impactos ecológicos, utilizando a tecnologia single-use com biorreatores onde foi demonstrado a sua eficácia em empresas de reciclagem.

\section{Fabricação dos biofármacos}

Os biofármacos vêm demonstrando uma necessidade no mercado Farmacêutico atual. Por se tratar de uma nova tecnologia de medicamentos os custos de produção são caros e demorados.

A produção de um anticorpo, exemplificando, a cultura celular é necessária que se faça in vitro ou in vivo no líquido ascítico no abdômen de animais. A partir disso, as células são transferidas em frascos contendo nutrientes fundamentais para seu desenvolvimento. Após o crescimento celular o material é movido para um biorreator, onde condições como temperatura, $\mathrm{pH}$, concentração de nutrientes e níveis de oxigênio são controlados e adequados para cada processo de produção (Salerno; Matsumoto; Ferraz, 2018).

Demonstrando excelentes resultados positivos no tratamento de algumas doenças que não eram eficazes em seus tratamentos convencionas como o câncer, com eficácia e segurança, tem aberto aos olhos do mundo e assim favorecendo o desenvolvimento do mercado de produção de biofármacos, entretanto, esse processo produtivo possui características que os tornam caras e demoradas, desta forma, faz necessário maiores investimentos financeiros em pesquisa e desenvolvimento (Salerno; Matsumoto; Ferraz, 2018).

\section{Impactos ecológicos}

Uma das tecnologias empregadas na fabricação dos biofármacos é o single-use, biorreatores utilizados com bolsas, e dispositivos descartáveis, onde a otimização da produção e flexibilidade do processo é uma de suas vantagens, já que as bolsas descartáveis não necessitam do processo de decomposição ou a retirada do material (Interfama, 2016). 
Outros benefícios podem ser levados em consideração com a utilização da tecnologia de single-use na produção de biofármacos, dentre os quais pode-se destacar: A facilidade operacional com a redução das etapas do processo; Eliminação dos custos com limpeza e tempo inativo da produção; Redução do consumo de água e energia, bem como a contaminação dos efluentes; Menor exposição do operador; O risco com contaminação cruzada é diminuído; E melhora os valores de conformidade, pois reduzem o potencial de erro e aumentam a produtividade (Whitford, 2010).

Nos Estado Unidos da América, hás empresas especializadas que fazem o processo de Reciclagem Biofarmacêutica, como a MilliporeSigma e a Triumvirate Environmenta, estas empresas reciclam o plástico inativado com potência nocivo para os mares e são reaproveitados para outros fins (Rader; Langer, 2018).

\section{Considerações Finais}

Os biofármacos demonstraram uma importante opção para o tratamento do câncer, uma nova alternativa é fundamental para encerrar de vez tratamento convencionais que acabam prejudicando de forma nociva o organismo do indivíduo. Com menos efeitos colaterais apresentados em testes sem dúvida esse será o futuro da Oncologia.

Discute-se também, o processo de fabricação complexo que acaba atrasando o avanço necessário para ampliar e quebrar ainda mais as barreiras do câncer, faz necessário maiores investimentos para acelerar o processo de novos biofármacos e maior acesso para países em desenvolvimento como o Brasil.

\section{Referências}

Brasil (2010). Anvisa. RDC N ${ }^{\circ}$ 55: Registro de produtos biológicos. Brasil, 16 de dezembro de 2010. 2010.Disponível em: <http://bvsms.saude.gov.br/bvs/saudelegis/ anvisa/2010/anexo/anexo_res0055_16_12_2010.p df >. Acesso em 18 de mar de 2020.

Brasil (2011). Instituto Nacional de Câncer José Alencar Gomes da Silva, INCA/Ministério da Saúde. ABC do câncer - Abordagens básicas para o controle do câncer. Rio de Janeiro 2011.Disponível em: https://bvs.saude.gov.br/bvs/publicacoes/inca/bc_do_cancer_2ed.pdf. Acesso em 18 de março de 2020.

Brasil (2017). Instituto Nacional de Câncer José Alencar Gomes da Silva, INCA/Ministério da Saúde. Definição de imunoterapia. 2017. Disponível em: http://www.inca.gov.br/conteudo_view.asp?ID=104. Acesso em 21 de março de 2020. 
Brasil (2018). ABDI. Agência Brasileira de Desenvolvimento Industrial. Biofármacos: Panorama Econômico. 2018. Disponível em :<http://ats.abdi.com.br/SiteAssets/ Panorama\%20Tecnologico\%20ATS \%20Biofarmacos\%20final.pdf $>$. Acesso em 18 de mar de 2020.

Castilho, 1. (2010). Biofármacos: Desenvolvimento atual. Rev Enifarmed, Rio de Janeiro, (3). Disponível em: http://ipd-farma.org.br/uploads/paginas/file/palestras/3_ENIFarMed /Leda_R_Castilho.pdf. Acesso em mar de 2020.

Delmondes, A. P. A., Coelho, J. L. G., Neves, S. A., Santana, W. J. de. (2020). Importância da Contribuição do Profissional Farmacêutico no Controle de Epidemias. Id on Line Rev.Mult.Psic., Julho/2020, vol.14, n.51, p. 408-412. ISSN: 1981-1179.

FERREIRA, L. et al (2014). Medicamentos Biológicos: Um caminho para a redução de toxicidades no tratamento do câncer. Rev. Sincifar, Belo Horizonte. Disponível em: http://hdl.handle.net/10419/211349. Acesso em 23 de mar de 2020.

Galluzzi, L. et al (2014). Classificação das imunoterapias anticâncer atuais. Alvo Onsegment, 5 (24), 12472.

Interfarma (2016). Biofármacos: A nova geração de insumos para a indústria brasileira. Rev Interfarma, São Paulo. Disponível em: https://www.interfarma.org.br/noticias/1020. Acesso em 23 de março de 2020

Oliveira, V. V., Silva, V. V. (2018). Biotecnologia para a produção de biofármacos: farmacovigilância, regulamentação e mercado no brasil 2018. Rev. Oswaldocruz, São Paulo. Disponível em: http://www.revista.oswaldocruz.br/Content/pdf/Edicao_19_Veridiana _Oliveira.pdf>. Acesso em: 18 mar 2020.

Rader, A., Langer, E (2018). Single-use bioprocessing equipment trends and adoption by cmos the growth in adoption of single-use systems for commercial manufacturing will be dramatic in coming years. Rev. Biopharm, 31(1):10-13. Disponível em: http://www.biopharminternational.com/singleuse-bioprocessing-equipment-trends-and-adoption-cmos. Acesso em 23 de março de 2020.

Salerno, M. S., Matsumoto, C., \& Ferraz, I. (2018). Biofármacos no Brasil: características, importância e delineamento de políticas públicas para seu desenvolvimento (No. 2398). Texto para Discussão.

Whitford, W. G (2010). Single-use systems as principais components in bioproduction. Rev. BioProcess Int, 8(11):34-42. Disponível em: https://bioprocessintl.com/upstream processing/upstreamsingle-use-technologies/single-use-systems-as-principal-components-inbioproduction-307214/,2010.

Acesso em 23 de março de 2020.

World Health Organisation/WHO (2016). Disponível em: http://www.who.int/cancer/en/. Acesso em 20 de março de 2020.

\section{How to cite this article (APA format):}

Franco Filho, João Francisco Lucena; Coelho, José Leonardo Gomes; Sousa, Carmelita Maria Silva; Fernandes, Elis Augusta Moura Dias; Brito, Rivaniery Teles Braga Emídio de; Santana, Willma José de (2020). Biopharmaceuticals: A New Alternative in Cancer Treatment?. Am. In. Mult. J., Jul to Out. (9) 5, 119-123. 